\title{
Topological phase separation in 2D hard-core Bose-Hubbard system away from half-filling
}

\author{
A.S. Moskvin, ${ }^{1}$ I.G. Bostrem, ${ }^{1}$ and A.S. Ovchinnikov ${ }^{1}$ \\ ${ }^{1}$ Ural State University, 620083, Ekaterinburg, Russia
}

(Dated: November 20, 2018)

\begin{abstract}
We suppose that the doping of the $2 \mathrm{D}$ hard-core boson system away from half-filling may result in the formation of multi-center topological defect such as charge order (CO) bubble domain(s) with Bose superfluid (BS) and extra bosons both localized in domain wall(s), or a topological $\mathrm{CO}+\mathrm{BS}$ phase separation, rather than an uniform mixed $\mathrm{CO}+\mathrm{BS}$ supersolid phase. Starting from the classical model we predict the properties of the respective quantum system. The long-wavelength behavior of the system is believed to remind that of granular superconductors, CDW materials, Wigner crystals, and multi-skyrmion system akin in a quantum Hall ferromagnetic state of a 2D electron gas.
\end{abstract}

One of the fundamental hot debated problems in bosonic physics concerns the evolution of the charge ordered $(\mathrm{CO})$ ground state of $2 \mathrm{D}$ hard-core Bose-Hubbard (BH) model (hc-BH) with a doping away from half-filling. Numerous model studies steadily confirmed the emergence of "supersolid" phases with simultaneous diagonal and off-diagonal long range order while Penrose and Onsager ${ }^{1}$ were the first showing as early as 1956 that supersolid phases do not occur.

The most recent quantum Monte-Carlo (QMC) simulations ${ }^{2.3 .4}$ found two significant features of the 2D BH model with a screened Coulomb repulsion: the absence of supersolid phase at half-filling, and a growing tendency to phase separation $(\mathrm{CO}+\mathrm{BS})$ upon doping away from half-filling. Moreover, Batrouni and Scalettar ${ }^{2}$ studied quantum phase transitions in the ground state of the 2D hc-BH Hamiltonian and have shown numerically that, contrary to the generally held belief, the most commonly discussed "checkerboard" supersolid is thermodynamically unstable and phase separates into solid and superfluid phases. The physics of the $\mathrm{CO}+\mathrm{BS}$ phase separation in $\mathrm{BH}$ model is associated with a rapid increase of the energy of a homogeneous $\mathrm{CO}$ state with doping away from half-filling due to a large "pseudo-spin-flip" energy cost. Hence, it appears to be energetically more favorable to "extract" extra bosons (holes) from the $\mathrm{CO}$ state and arrange them into finite clusters with a relatively small number of particles. Such a droplet scenario is believed to minimize the long-range Coulomb repulsion ${ }^{6}$ However, immediately there arise several questions. Whether a simple mean-field approximation (MFA) and classical continuum model can predict such a behavior? What is the detailed structure of the $\mathrm{CO}+\mathrm{BS}$ phase separated state? What are the main factors governing the essential low-energy and longwavelength physics? Is it possible to make use of simple toy models?

In the Letter we present a topological scenario of $\mathrm{CO}+\mathrm{BS}$ phase separation in 2D hc-BH model with intersite repulsion. The extra bosons/holes doped to a checkerboard CO phase of 2D boson system are believed to be confined in the ring-shaped domain wall of the skyrmion-like topological defect which looks like a bub- ble domain in an easy-axis antiferromagnet. This allows us to propose the mechanism of $2 \mathrm{D}$ topological $\mathrm{CO}+\mathrm{BS}$ phase separation when the doping of the bare checkerboard CO phase results in the formation of a multi-center topological defect, which simplified pseudo-spin pattern looks like a system of bubble CO domains with Bose superfluid confined in charged ring-shaped domain walls.

The Hamiltonian of hard-core Bose gas on a lattice can be written in a standard form as follows:

$H_{B G}=-\sum_{i>j} t_{i j} \hat{P}\left(B_{i}^{\dagger} B_{j}+B_{j}^{\dagger} B_{i}\right) \hat{P}+\sum_{i>j} V_{i j} N_{i} N_{j}-\mu \sum_{i} N_{i}$

where $\hat{P}$ is the projection operator which removes double occupancy of any site, $N_{i}=B_{i}^{\dagger} B_{i}, \mu$ the chemical potential determined from the condition of fixed full number of bosons $N_{l}=\sum_{i=1}^{N}\left\langle N_{i}\right\rangle$ or concentration $n=N_{l} / N \in[0,1]$. The $t_{i j}$ denotes an effective transfer integral, $V_{i j}$ is an intersite interaction between the bosons. Here $B^{\dagger}(B)$ are the Pauli creation (annihilation) operators which are Bose-like commuting for different sites $\left[B_{i}, B_{j}^{\dagger}\right]=0$, for $i \neq j$, while for the same site $B_{i}^{2}=\left(B_{i}^{\dagger}\right)^{2}=0$, $\left[B_{i}, B_{i}^{\dagger}\right]=1-2 N_{i}, N_{i}=B_{i}^{\dagger} B_{i} ; N$ is a full number of sites.

The model of hard-core Bose-gas with an intersite repulsion is equivalent to a system of spins $s=1 / 2$ exposed to an external magnetic field in the $z$-direction

$$
H_{B G}=\sum_{i>j} J_{i j}^{x y}\left(s_{i}^{+} s_{j}^{-}+s_{j}^{+} s_{i}^{-}\right)+\sum_{i>j} J_{i j}^{z} s_{i}^{z} s_{j}^{z}-h \sum_{i} s_{i}^{z},
$$

where $J_{i j}^{x y}=2 t_{i j}, J_{i j}^{z}=V_{i j}, h=\mu-\sum_{j(j \neq i)} V_{i j}, s^{-}=$ $\frac{1}{\sqrt{2}} B, s^{+}=-\frac{1}{\sqrt{2}} B^{\dagger}, s^{z}=-\frac{1}{2}+B_{i}^{\dagger} B_{i}, s^{ \pm}=\mp \frac{1}{\sqrt{2}}\left(s^{x} \pm \imath s^{y}\right)$.

In frames of of a conventional two-sublattice MFA approach one may introduce two vectors, the ferromagnetic and antiferromagnetic ones:

$$
\mathbf{m}=\frac{1}{2 s}\left(\left\langle\mathbf{s}_{1}\right\rangle+\left\langle\mathbf{s}_{2}\right\rangle\right) ; \mathbf{l}=\frac{1}{2 s}\left(\left\langle\mathbf{s}_{1}\right\rangle-\left\langle\mathbf{s}_{2}\right\rangle\right) ; \mathbf{m}^{2}+\mathbf{l}^{2}=1 .
$$

where $\mathbf{m} \cdot \mathbf{l}=0$. For the plane where these vectors lie one may introduce two-parametric angular de- 
scription: $m_{x}=\sin \alpha \cos \beta, m_{z}=-\sin \alpha \sin \beta, l_{x}=$ $\cos \alpha \sin \beta, l_{z}=\cos \alpha \cos \beta$. The hard-core boson system in a two-sublattice approximation is described by two diagonal order parameters $l_{z}, m_{z}$ and two complex off-diagonal order parameters $m_{ \pm}=\mp \frac{1}{\sqrt{2}}\left(m_{x} \pm \imath m_{y}\right)$ and $l_{ \pm}=\mp \frac{1}{\sqrt{2}}\left(l_{x} \pm \imath l_{y}\right)$. The complex superfluid order parameter $\Psi(\mathbf{r})=|\Psi(\mathbf{r})| \exp -\imath \varphi$ is determined by the in-plane components of ferromagnetic vector: $\Psi(\mathbf{r})=$ $\frac{1}{2}\left\langle\left(\hat{B}_{1}+\hat{B}_{2}\right)\right\rangle=s m_{-}=s m_{\perp} \exp -\imath \varphi, m_{\perp}$ being the length of the in-plane component of ferromagnetic vector. So, for a local condensate density we get $n_{s}=s^{2} m_{\perp}^{2}$. It is of interest to note that in fact all the conventional uniform $T=0$ states with nonzero $\Psi(\mathbf{r})$ imply simultaneous long-range order both for modulus $|\Psi(\mathbf{r})|$ and phase $\varphi$. The in-plane components of antiferromagnetic vector $l_{ \pm}$describe a staggered off-diagonal order. It is worth noting that by default one usually considers the negative sign of the transfer integral $t_{i j}$, that implies the ferromagnetic in-plane pseudospin ordering.

The model exhibits many fascinating quantum phases and phase transitions. Early investigations predict at $T=0$ charge order $(\mathrm{CO})$, Bose superfluid (BS) and mixed $(\mathrm{CO}+\mathrm{BS})$ supersolid uniform phases with an Ising-type melting transition (CO-NO) and KosterlitzThouless-type (BS-NO) phase transitions to a non-

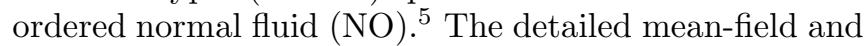
spin-wave analysis of the uniform phases of $2 \mathrm{D}$ hc- $\mathrm{BH}$ model is given by Pich and Frey $\underline{\underline{z}}$

MFA yields for the conventional uniform supersolid phase?

$$
\sin ^{2} \beta=m_{z} \frac{\sqrt{V-2 t}}{\sqrt{V+2 t}} ; \quad \sin ^{2} \alpha=m_{z} \frac{\sqrt{V+2 t}}{\sqrt{V-2 t}}
$$

with a constant chemical potential $\mu=4 s \sqrt{\left(V^{2}-4 t^{2}\right)}$. It should be noted that the supersolid phase appears to be unstable with regard to small perturbations in the Hamiltonian. The mean-field energy per site of the uniform supersolid phase is written as follows ${ }^{7}$

$$
E_{S S}=E_{C O}+s \mu m_{z}=E_{C O}+\mu\left(n_{B}-\frac{1}{2}\right),
$$

where $E_{C O}=-2 V s^{2}$. The cost of doping both for CO and $\mathrm{CO}+\mathrm{BS}$ phase appears to be rather high as compared with an easy-plane BS phase at half-filling where the chemical potential turns into zero $\underline{8}$

Magnetic analogy allows us to make unambiguous predictions as regards the doping of $\mathrm{BH}$ system away from half-filling. Indeed, the boson/hole doping of checkerboard $\mathrm{CO}$ phase corresponds to the magnetization of an antiferromagnet in $z$-direction. In the uniform easy-axis $l_{z}$-phase of anisotropic antiferromagnet the local spin-flip energy cost is rather big. In other words, the energy cost for boson/hole doping into checkerboard $\mathrm{CO}$ phase appears to be big due to a large contribution of boson-boson repulsion.

However, the magnetization of the anisotropic antiferromagnet in an easy axis direction may proceed as a first order phase transition with a "topological phase separation" due to the existence of antiphase domains $\frac{9}{9}$ The antiphase domain walls provide the natural nucleation centers for a spin-flop phase having enhanced magnetic susceptibility as compared with small if any longitudinal susceptibility thus providing the advantage of the field energy. Namely domain walls would specify the inhomogeneous magnetization pattern for such an anisotropic easy-axis antiferromagnet in relatively weak external magnetic field. As concerns the domain type in quasi-two-dimensional antiferromagnet one should emphasize the specific role played by the cylindrical, or bubble domains which have finite energy and size. These topological solitons have the vortex-like in-plane spin structure and often named "skyrmions". The classical, or Belavin-Polyakov (BP) skyrmion ${ }^{10}$ describes the solutions of a non-linear $\sigma$-model with a classical isotropic $2 \mathrm{D}$ Heisenberg Hamiltonian and represents one of the generic toy model spin textures. It is of primary importance to note that skyrmion-like bubble domains in easy-axis layered antiferromagnets were actually observed in the experiments performed by Waldner, 11 that were supported later by different authors (see, e.g. Refs 12 13). Although some questions were not completely clarified and remain open until now $\stackrel{14.15}{\longleftarrow}$ the classical and quantum ${ }^{16}$ skyrmion-like topological defects are believed to be a genuine element of essential physics both of ferro- and antiferromagnetic $2 \mathrm{D}$ easy-axis systems.

The magnetic analogy seems to be a little bit naive, however, it catches the essential physics of doping the hc-BH system. As regards the checkerboard $\mathrm{CO}$ phase of such a system, namely a finite energy skyrmion-like bubble domain ${ }^{17}$ seems to be the most preferable candidate for the domain with antiphase domain wall providing the natural reservoir for extra bosons. The classical description of nonlinear excitations in hc-BH model includes the skyrmionic solution given $V=2 t \underline{17}$ The skyrmion spin texture consists of a vortex-like arrangement of the inplane components of ferromagnetic $\mathbf{m}$ vector with the $l_{z^{-}}$ component reversed in the centre of the skyrmion and gradually increasing to match the homogeneous background at infinity. The simplest spin distribution within classical skyrmion is given as follows

$$
\begin{gathered}
m_{x}=m_{\perp} \cos \left(\varphi+\varphi_{0}\right) ; m_{y}=m_{\perp} \sin \left(\varphi+\varphi_{0}\right) ; \\
m_{\perp}=\frac{2 r \lambda}{r^{2}+\lambda^{2}} ; l_{z}=\frac{r^{2}-\lambda^{2}}{r^{2}+\lambda^{2}},
\end{gathered}
$$

where $\varphi_{0}$ is a global phase $(U(1)$ order parameter $), \lambda$ skyrmion radius. The skyrmion spin texture describes the coexistence and competition of the charge order parameter $l_{z}$ and $\mathrm{BS}$ order parameter $m_{\perp}$ that reflects a complex spatial interplay of potential and kinetic energies. The skyrmion looks like a bubble domain in an easy-axis magnet. Skyrmions are characterized by the magnitude and sign of its topological charge, by its size (radius), and by the global orientation of the spin. The 
scale invariance of classical BP skyrmionic solution reflects in that its energy does not depend on radius, and global phase. An interesting example of topological inhomogeneity is provided by a multi-center BP skyrmion ${ }^{10}$ which energy does not depend on the position of the centers. The latter are believed to be addressed as an additional degree of freedom, or positional order parameter.

It should be noted that the domain wall in such a bubble domain somehow created in the checkerboard $\mathrm{CO}$ phase of $2 \mathrm{D}$ hc-BH system represents an effective ringshaped reservoir both for Bose-superfluid and extra boson/hole. Indeed, the magnetic response of a fixed radius skyrmion to a weak longitudinal external magnetic field can be approximated as follows:

$$
m_{z}(r) \approx \chi(r) h,
$$

where $\chi(r)=f(r) m_{\perp}(r)$ is a local magnetic susceptibility, and $f(r)$ has distinct maximum at the domain wall center $r=\lambda$ with oscillating behavior $f(r) \propto J_{1}(r \sqrt{2}) \propto$ $\sin (r \sqrt{2}+\pi / 4) / \sqrt{r}$ for large $r$. The oscillating character of the magnetization distribution seems to reflect a skyrmion instability. Thus we conclude that the extra boson/hole density described by the order parameter $m_{z}$ is to be localized in the domain wall. This result is supported by numerical calculations for the static skyrmionlike excitations in a continuous 2D hc-BH model with a strong boson-boson repulsion that was considered by us earlier in Ref 17 . The Fig. 1 sketches the calculated radial distribution of the order parameter $m_{z}$ for a single bubble domain in hc-BH system with injected small boson concentration away from half-filling. It appears to be trapped inside the domain wall. As expected, the soliton energy depends quadratically on the number $\Delta n$ of bosons bound in domain wall (see insert in Fig. 1), similarly to that of homogeneous BS phase $\stackrel{8}{*}$ In other words, $\partial E / \partial n=0$ and one might say about a zero value of the effective boson/hole chemical potential for the CO bubble domain configuration would it be a ground state.

In the continuous model the classical BP skyrmion is a topological excitation and cannot dissipate. However, the classical static skyrmion is unstable with regard to an external field, anisotropic interactions both of easy-plane and easy-axis type. Small easy-axis anisotropy or external field are sufficient to shrink skyrmion to a nanoscopic size when magnetic length $l_{0}$ :

$$
l_{0}=\left(\sqrt{(2 V / t)^{2}-(\mu / t)^{2}}-4\right)^{-\frac{1}{2}}
$$

is of the order of several lattice parameters, and the continuous approximation fails to correctly describe excitations. Nonetheless, Abanov and Pokrovsky 18 have shown that the easy-axis anisotropy together with fourthorder exchange term can stabilize skyrmion with radius $R \propto \sqrt{l_{0}}$.

A skyrmionic scenario in hc-BH model allows us to make several important predictions. Away from halffilling one may anticipate the nucleation of a topological defect in the unconventional form of the multi-center

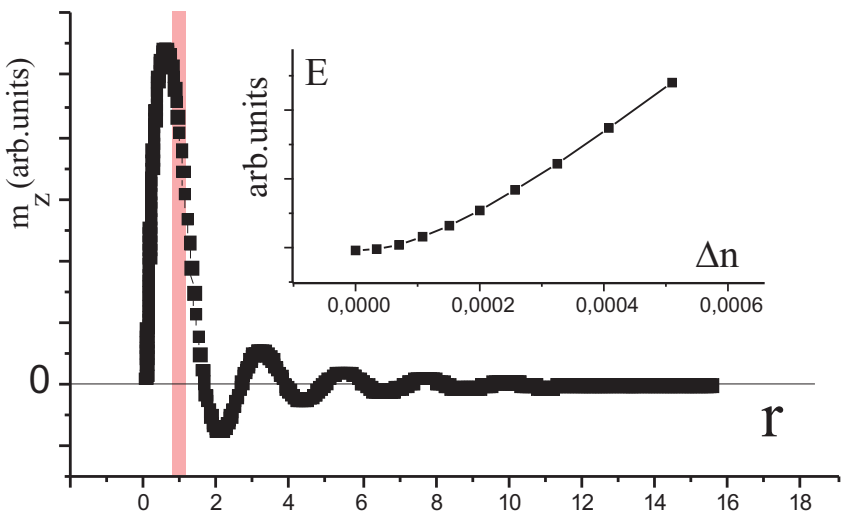

FIG. 1: The results of numerical calculations by shooting method for a shrinked skyrmion given $v=2.1, t=1$ : main panel - the radial distribution of the order parameter $m_{z}$ (the filled region points to a center of a domain wall); the insert shows the dependence of the soliton energy on the number $\Delta n$ of bosons bound in a domain wall.

skyrmion-like object with ring-shaped Bose superfluid regions positioned in an antiphase domain wall separating the $\mathrm{CO}$ core and $\mathrm{CO}$ outside of the single skyrmion. The specific spatial separation of BS and CO order parameters that avoid each other reflects the competition of kinetic and potential energy. Such a topological $(\mathrm{CO}+\mathrm{BS})$ phase separation is believed to provide a minimization of the total energy as compared with its uniform supersolid counterpart. Thus, the parent checkerboard CO phase may gradually loose its stability under boson/hole doping, while a novel topological self-organized texture is believed to become stable. The most probable possibility is that every domain wall accumulates single boson, or boson hole. Then, the number of centers in a multicenter skyrmion nucleated with doping has to be equal to the number of bosons/holes. In such a case, we anticipate the near-linear dependence of the total BS volume fraction on the doping. Generally speaking, one may assume scenario when the nucleation of a multicenter skyrmion occurs spontaneously with no doping. In such a case we should anticipate the existence of neutral multi-center skyrmion-like object with equal number of positively and negatively charged single skyrmions. However, in practice, namely the boson/hole doping is likely to be a physically clear driving force for a nucleation of a single, or multi-center skyrmion-like self-organized collective mode in the form of multi-center charged topological defect which may be (not strictly correctly) referred to as multi-skyrmion system akin in a quantum Hall ferromagnetic state of a two-dimensional electron gas. $\stackrel{19}{=}$ In such a case, we may characterize an individual skyrmion by its position (i.e., the center of skyrmionic texture), its size (i.e., the radius of domain wall), and the orientation of the in-plane components of pseudo-spin (U(1) degree of freedom). An isolated skyrmion is described by the inhomogeneous distribution of the CO parameter, or staggered boson density $l_{z}$, order parameter $m_{z}$ charac- 
terizing the deviation from the half-filling, and $m_{\perp}$ that corresponds only to the modulus of the superfluid order parameter.

It seems likely that for a light doping any doped particle (boson/holes) results in a nucleation of a new singleskyrmion state, hence its density changes gradually with particle doping. Therefore, as long as the separation between skyrmionic centers is sufficiently large so that the inter-skyrmion interaction is negligible, the energy of the system per particle remains almost constant. This means that the chemical potential of boson or hole remains unchanged with doping and hence apparently remains fixed.

The multi-skyrmionic system in contrast with uniform ones can form the structures with inhomogeneous longrange ordering of the modulus of the superfluid order parameter accompanied by the unordered global phases of single skyrmions. Such a situation resembles in part that of granular superconductivity.

In the long-wavelength limit the off-diagonal ordering can be described by an effective Hamiltonian in terms of U(1) (phase) degree of freedom associated with each skyrmion. Such a Hamiltonian contains a repulsive, longrange Coulomb part and a short-range contribution related to the phase degree of freedom. The latter term can be written out in the standard for the $X Y$ model form of a so-called Josephson coupling

$$
H_{J}=-\sum_{\langle i, j\rangle} J_{i j} \cos \left(\varphi_{i}-\varphi_{j}\right),
$$

where $\varphi_{i}, \varphi_{j}$ are global phases for skyrmions centered at points $i, j$, respectively, $J_{i j}$ Josephson coupling parameter. Namely the Josephson coupling gives rise to the long-range ordering of the phase of the superfluid order parameter in a multi-center skyrmion. Such a Hamiltonian represents a starting point for the analysis of disordered superconductors, granular superconductivity, insulator-superconductor transition with $\langle i, j\rangle$ array of superconducting islands with phases $\varphi_{i}, \varphi_{j}$. Calculating the phase-dependent part of skyrmion-skyrmion interaction Timm et al ${ }^{20}$ arrived at negative sign of $J_{i j}$ that favors antiparallel alignment of the U(1) pseudospins. In other words, two skyrmions are assumed to form a peculiar Josephson $\pi$ micro-junction. There are a number of interesting implications that follow directly from this result: ${ }^{21}$ the spontaneous breaking of time-reversal symmetry with non-zero supercurrents and magnetic fluxes in the ground state, long-time tails in the dynamics of the system, unconventional Aharonov-Bohm period $h c / 4 e$, negative magnetoresistence.

To account for Coulomb interaction and allow for quantum corrections we should introduce into effective Hamiltonian the charging energy $y^{21}$

$$
H_{c h}=-\frac{1}{2} q^{2} \sum_{i, j} n_{i}\left(C^{-1}\right)_{i j} n_{j},
$$

where $n_{i}$ is a boson number operator for bosons bound in $i$-th skyrmion; it is quantum-mechanically conjugated to $\varphi: n_{i}=-i \partial / \partial \varphi_{i},\left(C^{-1}\right)_{i j}$ stands for the capacitance matrix, $q$ for bosonic charge.

Such a system appears to reveal a tremendously rich quantum-critical structure. In the absence of disorder, the $T=0$ phase diagram of the multi-skyrmion system implies either triangular, or square crystalline arrangements (Skyrmion crystal) with possible melting transition to a Skyrmion liquid. It should be noted that analogy with charged $2 D$ Coulomb gas implies the Wigner crystallization of multi-center skyrmion with Wigner crystal to Wigner liquid melting transition, respectively. Naturally, that the additional degrees of freedom for skyrmion provide a richer physics of Skyrmion lattices. For a WC to be an insulator, disorder is required, which pins the WC and also causes the crystalline order to have a finite correlation length. Traditional approach to a Wigner crystallization implies the formation of a WC for densities lower than a critical density, when the Coulomb energy dominates over the kinetic energy. The effect of quantum fluctuations leads to a (quantum) melting of the solid at high densities, or at a critical lattice spacing. The critical properties of a two-dimensional lattice without any internal degree of freedom are successfully described applying the BKT theory to dislocations and disclinations of the lattice, and proceeds in two steps. The first implies the transition to a liquid-crystal phase with short-range translational order, the second does the transition to isotropic liquid. Disorder pins the Skyrmion lattice and also causes the crystalline order to have a finite correlation length. For such a system provided the skyrmion positions fixed at all temperatures, the longwave-length physics would be described by an antiferromagnetic $X Y$ model with expectable BKT transition and gapless $X Y$ spin-wave mode.

As regards the superfluid properties the skyrmionic liquid reveals unconventional behavior with two critical temperatures $T_{B S} \leq t$ and $T_{c} \leq J, T_{B S}$ being the temperature of the ordering of the modulus and $T_{c}<T_{B S}$ that of the phase of order parameter $\Psi$.

The low temperature physics in Skyrmion crystals is governed by an interplay of two BKT transitions, for the $\mathrm{U}(1)$ phase and positional degrees of freedom, respectively ${ }_{20}$ Dislocations in most Skyrmion lattice types lead to a mismatch in the U(1) degree of freedom, which makes the dislocations bind fractional vortices and lead to a coupling of translational and phase excitations. Both BKT temperatures either coincide (square lattice) or the melting one is higher (triangular lattice) $\underline{20}^{20}$

Quantum fluctuations can substantially affect these results. Quantum melting can destroy U(1) order at sufficiently low densities where the Josephson coupling becomes exponentially small. Similar situation is expected to take place in the vicinity of structural transitions in Skyrmion crystal. With increasing the skyrmion density the quantum effects result in a significant lowering of the melting temperature as compared with classical squareroot dependence. The resulting melting temperature can reveal an oscilating behavior as a function of particle den- 
sity with zeros at the critical (magic) densities associated with structural phase transitions.

In terms of our model, the positional order corresponds to an incommensurate charge density wave, while the $\mathrm{U}(1)$ order does to a superconductivity. In other words, we arrive at a subtle interplay between two orders. The superconducting state evolves from a charge order with $T_{C} \leq T_{m}$, where $T_{m}$ is the temperature of a melting transition which could be termed as a temperature of the opening of the insulating gap (pseudo-gap!?).

The normal modes of a dilute skyrmion system (multicenter skyrmion) include the pseudo-spin waves propagating in-between the skyrmions, the positional fluctuations, or phonon modes, of the skyrmions which are gapless in a pure system, but gapped when the lattice is pinned, and, finally, fluctuations in the skyrmionic inplane orientation and size. The latter two types of fluctuation are intimitely connected, since the $z$-component of spin and orientation are conjugate coordinates because of commutation relations of quantum angular momentum operators. So, rotating a skyrmion changes its size. The orientational fluctuations of the multi-skyrmion system are governed by the gapless $X Y$ model ${ }^{19}$ The relevant model description is most familiar as an effective theory of the Josephson junction array. An important feature of the model is that it displays a quantum-critical point.

The low-energy collective excitations of skyrmion liquid includes an usual longitudinal acoustic phonon branch. The liquid crystal phases differ from the isotropic liquid in that they have massive topological excitations, i.e., the disclinations. One should note that the liquids do not support transverse modes, these could survive in a liquid state only as overdamped modes. So that it is reasonable to assume that solidification of the skyrmion lattice would be accompanied by a stabilization of transverse modes with its sharpening below melting transition. In other words an instability of transverse phonon modes signals the onset of melting.

A generic property of the positionally ordered skyrmion configuration is the sliding mode which is usually pinned by the disorder. The depinning of sliding mode(s) can be detected in a low-frequency and lowtemperature optical response.

In conclusion, the boson/hole doping of the hard-core boson system away from half-filling is assumed to be a driving force for a nucleation of a multi-center skyrmionlike self-organized collective mode that resembles a system of CO bubble domains with a Bose superfluid and extra bosons both confined in domain walls. Such a topological $\mathrm{CO}+\mathrm{BS}$ phase separation, rather than an uniform mixed $\mathrm{CO}+\mathrm{BS}$ supersolid phase, is believed to describe the evolution of hc-BH model away from halffilling. Starting from the classical model we predict the properties of the respective quantum system. In frames of our scenario we may anticipate for the hc-BH model the emergence of an inhomogeneous BS condensate for superhigh temperatures $T_{T P S} \leq t$, and $3 \mathrm{D}$ superconductivity for rather high temperatures $T_{c} \leq J<t$. The system is believed to reveal many properties typical for granular superconductors, CDW materials, Wigner crystals, and multi-skyrmion system akin in a quantum Hall ferromagnetic state of a 2D electron gas. Topological inhomogeneity is believed to be a generic property of $2 \mathrm{D}$ hard-core boson systems away from half-filling.

Despite all shortcomings, MFA and continuous approximation are expected to provide a physically clear semiquantitative picture of rather complex transformations taking place in bare $\mathrm{CO}$ system with doping, and can be instructive as a starting point to analyze possible scenarios. First of all, the MFA analysis allow us to consider the antiphase domain wall in CO phase to be a very efficient ring-shaped potential well for the localization of a single extra boson (hole) thus forming a novel type of a topological defect with a single-charged domain wall. Such a defect can be addressed as a charged skyrmionlike quasiparticle which energy can be approximated by its classical value for $\mathrm{CO}$ bubble domain. It is of great importance to note that domain wall simultaneously represents a ring-shaped reservoir for Bose superfluid.

Unfortunately, we have no experience to deal with multi-center skyrmions as regards its structure, energetics, and stability. It should be noted that such a texture with strongly polarizable centers is believed to provide an effective screening of long-range boson-boson repulsion thus resulting in an additional self-stabilization. Nucleation of topological phase is likely to proceed in the way typical for the first order phase transitions. The present paper establishes only the framework for analyzing the subtleties of the phase separation in a lattice hc-BH model away from half-filling. Much work remains to be done both in a macroscopic and microscopic aproaches.

We acknowledge the support by SMWK Grant, INTAS Grant No. 01-0654, CRDF Grant No. REC-005, RME Grant No. E 02-3.4-392 and No. UR.01.01.042, RFBR Grant No. 01-02-96404. One of us (A.S.M.) has benefited from discussions with C. Timm, S.-L. Drechsler, and T. Mishonov.
1 O. Penrose and L. Onsager, Phys. Rev. 104, 576 (1956).

2 G. G. Batrouni and R. T. Scalettar, Phys. Rev. Lett. 84, 1599 (2000).

3 F. Hébert, G.G. Batrouni, R.T. Scalettar et. al., Phys. Rev. B 65, 014513 (2001)

${ }^{4}$ Guido Schmid, Synge Todo, Matthias Troyer, and Ansgar
Dorneich, Phys. Rev. Lett. 88, 167208 (2002).

5 R.T. Scalettar, G.G. Batrouni, A.P. Kampf, and G.T. Zimanyi, Phys. Rev. B 51, 8467 (1995).

6 M.Yu. Kagan, K.I. Kugel, and D.I. Khomskii, JETP, 93, 415 (2001).

7 Christian Pich and Erwin Frey, Phys. Rev. B 57, 13712 
(1998).

8 K. Bernardet, G.G. Batrouni, J.-L. Meunier et. al., Phys. Rev. B 65, 104519 (2002).

9 The antiferromagnetic domain texture appears as a result of the minimization of elastic and magnetoelastic energies.

10 A.A. Belavin, A.M. Polyakov, JETP Lett. 22, 245 (1975).

11 F. Waldner, J. Magn. Mag. Matter 54-57, 837 (1986); Phys. Rev. Lett. 65, 1519 (1990).

12 S.I. Belov, B.I. Kochelaev, Sol. St. Commun. 103, 249 (1997).

13 Carsten Timm and K. H. Bennemann, Phys. Rev. Lett. 84, 4994 (2000).

14 T. Kamppeter, S.A. Leonel, F.G. Mertens, M.E. Gouva, A.S.T. Pires, and A.S. Kovalev, Eur. Phys. J. B 21, 93 (2001).
15 Denis D. Sheka, Boris A. Ivanov, and G. Mertens, Phys. Rev. B 64, 024432 (2001).

16 R.A. Istomin, A.S. Moskvin, JETP Lett. 61, 898 (2000).

17 A.S. Ovchinnikov, I.G. Bostrem, A.S. Moskvin, Phys. Rev. B 66, 134304 (2002).

18 Ar. Abanov, V.L. Pokrovsky, Phys. Rev. B 58, R8889 (1998).

19 A.G. Green, Phys. Rev. B 61, R16299 (2000).

20 Carsten Timm, S.M. Girvin, H.A. Fertig, Phys. Rev. B 58, 10634 (1998)

21 S.A. Kivelson, B.Z. Spivak, Phys. Rev. B 45, 10490 (1992).

${ }^{22}$ Madan Rao, Surajit Sengupta, and R. Shankar, Phys. Rev. Lett. 79, 3998 (1997). 